\section{FREE INTRODUCTORY IMPLANT PACK}

General dental practitioners (GDPs) can now restore the most common implant cases. Whether a patient has one, several or all of their teeth missing, their own dentist can complete the treatment following implant placement.

Dentsply Friadent's 'R£LAX' package offers free training to help GDPs get started in dental implant restoration. The programme has been expanded to include removable implant-supported full arch acrylic bridges.

Increasing numbers of GDPs are discovering that restoring implant cases can be good for the practice and can enhance long-term patient relationships. The R£LAX package includes three hours of CPD and a free mini prosthetics kit. Further details and a full list of forthcoming local events are available on www.dentists4implants.com.

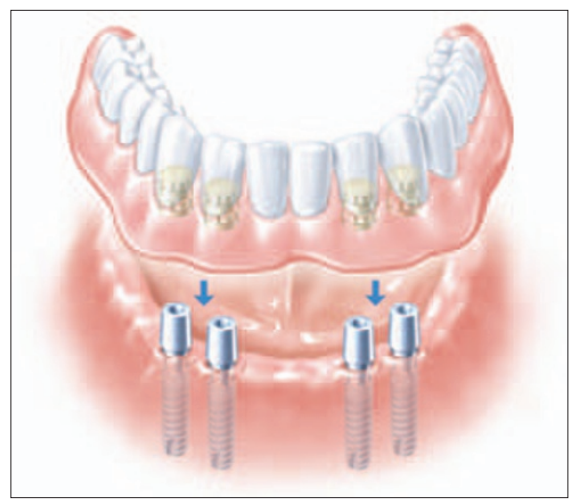

\section{IMPLANT COURSE DATE CHANGE}

The Implantology Year Course with Dr David Guy, in association with Biohorizons, comprises ten lectures over 12 months, as well as practical sessions, and will now commence in March 2012.

Offering 70 hours of verifiable CPD and requiring no experience of implantology, this course provides a very high level of training, enabling clinicians to perform implant dentistry skilfully and with confidence.

The lectures and practical sessions allowing each delegate to treat up to ten of their own patients under one-to-one supervision - will be held at Dr Guy's practice in Cardiff.

The Implantology Year Course costs $£ 4,000$ per person and is limited to eight participants.

For further details visit www. courses.guysdentalclinic.co.uk, email courses@guysdentalclinic.co.uk or call 02920220794.

\section{BEST KEPT SECRET}

Since its benefits first came to light during the sugar shortages of the Second World War, xylitol has long been one of the 'best kept secrets' in the fight against tooth decay, and is now gaining increasing scientific recognition as a powerful means of improving oral health.

Xylitol is a naturally occurring white crystalline sugar alcohol that looks and tastes like normal sugar but with 40\% fewer calories. While it has significant general benefits, xylitol is also scientifically proven to help prevent tooth decay and improve oral health. This is because xylitol has a unique chemical composition that is unfavourable to the metabolism of the bacteria most closely associated with tooth decay.
As a leading manufacturer of xylitol-based products, Spry produces a range of dentifrices, mints and gums that are perfect adjuncts to any comprehensive oral hygiene regime. These products include Spry Fluoride Toothpaste, Spry Xlear Oral Rinse and Spry Chewing Gum, which is available in a selection of interesting flavours including peppermint, fresh fruit, green tea and cinnamon.

For further information contact Anyone 4 Tea Ltd on 01730890290 or visit www.anyone4tea.com.

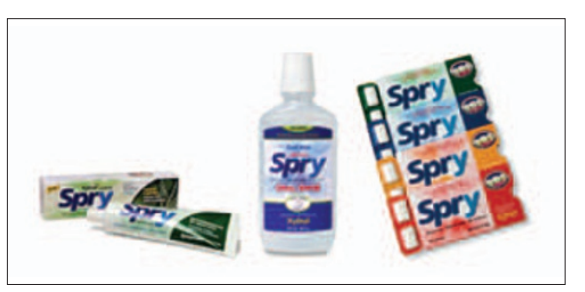

